\title{
Stability of Standing Waves for Nonlinear Schrödinger Equations with Inhomogeneous Nonlinearities
}

\author{
Anne De Bouard and Reika Fukuizumi
}

\begin{abstract}
The effect of inhomogeneity of nonlinear medium is discussed concerning the stability of standing waves $e^{i \omega t} \phi_{\omega}(x)$ for a nonlinear Schrödinger equation with an inhomogeneous nonlinearity $V(x)|u|^{p-1} u$, where $V(x)$ is proportional to the electron density. Here, $\omega>0$ and $\phi_{\omega}(x)$ is a ground state of the stationary problem. When $V(x)$ behaves like $|x|^{-b}$ at infinity, where $0<b<2$, we show that $e^{i \omega t} \phi_{\omega}(x)$ is stable for $p<1+(4-2 b) / n$ and sufficiently small $\omega>0$. The main point of this paper is to analyze the linearized operator at standing wave solution for the case of $V(x)=|x|^{-b}$. Then, this analysis yields a stability result for the case of more general, inhomogeneous $V(x)$ by a certain perturbation method.
\end{abstract}

\section{Introduction}

The nonlinear Schrödinger equations

$$
i \partial_{t} u=-\Delta u-g\left(x,|u|^{2}\right) u, \quad(t, x) \in \mathbb{R}^{1+n}
$$

arise in various physical contexts such as nonlinear optics and plasma physics. When $g\left(x,|u|^{2}\right)=V(x)|u|^{p-1}$, equation (1.1) can model beam propagation in an inhomogeneous medium where $V(x)$ is proportional to the electron density. L. Bergé [2] studied formally the stability condition for soliton solutions of the above type of equations, depending on the shape of $g\left(x,|u|^{2}\right)$. The real function $g\left(x,|u|^{2}\right)$ is a potential which can either stand for corrections to the nonlinear power-law response, or for some inhomogeneities in the medium. In addition, Towers and Malomed [29] recently observed by means of variational approximation and direct simulations that a certain type of time-dependent nonlinear medium gives rise to completely stable beams.

Akhmediev [1], Jones [17] and Grillakis, Shatah and Strauss [13] studied the existence and stability of solitary waves of (1.1) when $g\left(x,|u|^{2}\right)$ describes three layered media where the outside two are nonlinear and the sandwiched one is linear. Also, Merle [23] investigated the existence and nonexistence of blowup solutions of (1.1) for inhomogeneities of the form $g\left(x,|u|^{2}\right)=V(x)|u|^{4 / n}$.

In this paper, we will not exactly deal with the same nonlinearity as those in $[2,29]$, we consider the case $g\left(x,|u|^{2}\right)=V(x)|u|^{p-1}$ with $V(x)$ satisfying the following assumptions (V1) and (V2) with $n \geq 3,0<b<2$ and $1<p<$ $1+(4-2 b) /(n-2)$. 
(V1) $\quad V(x) \geq 0, \quad V(x) \not \equiv 0, \quad V(x) \in C\left(\mathbb{R}^{n} \backslash\{0\}, \mathbb{R}\right), \quad V(x) \in L^{\theta^{*}}(|x| \leq 1)$, where $\theta^{*}=2 n /\{(n+2)-(n-2) p\}$.

(V2) There exist $C>0$ and $a>\{(n+2)-(n-2) p\} / 2>b$ such that

$$
\left|\left(V(x)-\frac{1}{|x|^{b}}\right)\right| \leq \frac{C}{|x|^{a}}
$$

for all $x$ with $|x| \geq 1$.

The main purpose in this paper is to show that under the above assumptions on $V(x)$, the standing wave solution of (1.1) is stable for $p<1+(4-2 b) / n$ and sufficiently small frequency. As an example satisfying (V1) and (V2), we keep $V(x)=\left(1+|x|^{2}\right)^{-b / 2}$ in mind.

By a standing wave, we mean a solution of (1.1) of the form

$$
u_{\omega}(t, x)=e^{i \omega t} \phi_{\omega}(x)
$$

where $\omega>0$ and $\phi_{\omega}(x)$ is a ground state of the following stationary problem

$$
\left\{\begin{array}{l}
-\Delta \phi+\omega \phi-V(x)|\phi|^{p-1} \phi=0, \quad x \in \mathbb{R}^{n}, \\
\phi \in H^{1}\left(\mathbb{R}^{n}\right), \quad \phi \neq 0 .
\end{array}\right.
$$

We recall previous results. Several authors have been studying the problem of stability and instability of standing waves for (1.1) (see, e.g., [3, 6, 7, 9, 11, 13, $22,25,30,32])$. First, we consider the case $V(x) \equiv 1$, namely,

$$
i \partial_{t} u=-\Delta u-|u|^{p-1} u, \quad(t, x) \in \mathbb{R}^{1+n},
$$

where $1<p<\infty$ if $n=1,2$, and $1<p<1+4 /(n-2)$ if $n \geq 3$.

For $\omega>0$, there exists a unique positive radial solution $\psi_{\omega}(x)$ of

$$
\left\{\begin{array}{l}
-\Delta \psi+\omega \psi-|\psi|^{p-1} \psi=0, \quad x \in \mathbb{R}^{n}, \\
\psi \in H^{1}\left(\mathbb{R}^{n}\right), \quad \psi \neq \equiv 0 .
\end{array}\right.
$$

(See Strauss [26] and Berestycki and Lions [4] for the existence, and Kwong [19] for the uniqueness). It is known that a positive solution of (1.4) is a ground state. In [6] Cazenave and Lions proved that if $p<1+4 / n$ then the standing wave solution $e^{i \omega t} \psi_{\omega}(x)$ is stable for any $\omega>0$. On the other hand, it is shown that if $p \geq 1+4 / n$ then the standing wave solution $e^{i \omega t} \psi_{\omega}(x)$ is unstable for any $\omega>0$ (see Berestycki and Cazenave [3] for $p>1+4 / n$, and Weinstein [30] for $p=1+4 / n$ ). The aim of the paper is to study, in the case where $V(x)$ satisfies (V1) and (V2), what happens in the complementary case of the result in [11], where instability of standing waves was shown for $p>1+(4-2 b) / n$ and sufficiently small $\omega>0$.

We define the energy functional $E$ and the charge $Q$ on $H^{1}\left(\mathbb{R}^{n}\right)$ by

$$
E(v):=\frac{1}{2}\|\nabla v\|_{2}^{2}-\frac{1}{p+1} \int_{\mathbb{R}^{n}} V(x)|v(x)|^{p+1} d x, \quad Q(v):=\frac{1}{2}\|v\|_{2}^{2} .
$$


We remark that by the assumptions (V1) and (V2), the functional $E$ is well defined on $H^{1}\left(\mathbb{R}^{n}\right)$ if $p<1+(4-2 b) /(n-2)$.

The time local well-posedness for the Cauchy problem to (1.1) with $g\left(x,|u|^{2}\right)$ $=V(x)|u|^{p-1}$ in $H^{1}\left(\mathbb{R}^{n}\right)$ and the conservation of energy and charge hold (see, e.g., Theorem 4.4.6 of Cazenave [5]). Exactly, we have the following proposition.

Proposition 1 Let $n \geq 3$ and $1<p<1+(4-2 b) /(n-2)$. Assume (V1) and $\lim _{|x| \rightarrow \infty} V(x)=0$. Then, for any $u_{0} \in H^{1}\left(\mathbb{R}^{n}\right)$ there exist $T=T\left(\left\|u_{0}\right\|_{H^{1}}\right)>0$ and a unique solution $u(t) \in C\left([0, T], H^{1}\left(\mathbb{R}^{n}\right)\right)$ of $(1.1)$ with $u(0)=u_{0}$ satisfying

$$
E(u(t))=E\left(u_{0}\right), \quad Q(u(t))=Q\left(u_{0}\right), \quad t \in[0, T] .
$$

Before we state our theorem, we give some precise definitions.

Definition 1 For $\omega>0$, we define two functionals on $H^{1}\left(\mathbb{R}^{n}\right)$ :

$$
\begin{aligned}
& S_{\omega}(v):=E(v)+\omega Q(v) \quad \text { (action), } \\
& I_{\omega}(v):=\|\nabla v\|_{2}^{2}+\omega\|v\|_{2}^{2}-\int_{\mathbb{R}^{n}} V(x)|v(x)|^{p+1} d x .
\end{aligned}
$$

Let $\mathcal{G}_{\omega}$ be the set of all non-negative minimizers for

$$
\inf \left\{S_{\omega}(v): v \in H^{1}\left(\mathbb{R}^{n}\right) \backslash\{0\}, I_{\omega}(v)=0\right\} .
$$

The existence of non-negative minimizers for (1.5) was proved by the standard variational argument since $V(x)$ vanishes as $|x| \rightarrow \infty$ (see [26, 11]). Namely, we have

Lemma 1.1 Let $n \geq 3$ and $1<p<1+(4-2 b) /(n-2)$. Assume (V1) and $\lim _{|x| \rightarrow \infty} V(x)=0$. Then $\mathcal{G}_{\omega}$ is not empty for $\omega>0$.

\section{Remark 1.1}

(i) We note that

$$
I_{\omega}(v)=\left.\partial_{\lambda} S_{\omega}(\lambda v)\right|_{\lambda=1}=\left\langle S_{\omega}^{\prime}(v), v\right\rangle .
$$

(ii) Let $\phi_{\omega} \in \mathcal{G}_{\omega}$. Then, there exists a Lagrange multiplier $\Lambda \in \mathbb{R}$ such that $S_{\omega}^{\prime}\left(\phi_{\omega}\right)=\Lambda I_{\omega}^{\prime}\left(\phi_{\omega}\right)$. Thus, we have $\left\langle S_{\omega}^{\prime}\left(\phi_{\omega}\right), \phi_{\omega}\right\rangle=\Lambda\left\langle I_{\omega}^{\prime}\left(\phi_{\omega}\right), \phi_{\omega}\right\rangle$. Since

$$
\left\langle S_{\omega}^{\prime}\left(\phi_{\omega}\right), \phi_{\omega}\right\rangle=I_{\omega}\left(\phi_{\omega}\right)=0
$$

and

$$
\left\langle I_{\omega}^{\prime}\left(\phi_{\omega}\right), \phi_{\omega}\right\rangle=-(p-1) \int V(x)\left|\phi_{\omega}\right|^{p+1}<0,
$$

we have $\Lambda=0$. Namely, $\phi_{\omega}$ satisfies (1.2). Moreover, for any $v \in H^{1}\left(\mathbb{R}^{n}\right) \backslash\{0\}$ satisfying $S_{\omega}^{\prime}(v)=0$, we have $I_{\omega}(v)=0$. Thus, by the definition of $\mathcal{G}_{\omega}$, we have $S_{\omega}\left(\phi_{\omega}\right) \leq S_{\omega}(v)$. Namely, $\phi_{\omega} \in \mathcal{G}_{\omega}$ is a ground state (minimal action solution) of (1.2) in $H^{1}\left(\mathbb{R}^{n}\right)$. It is easy to see that a ground state of (1.2) in $H^{1}\left(\mathbb{R}^{n}\right)$ is a minimizer of $(1.5)$. 
The stability and instability in this paper is defined as follows.

Definition 2 For $\phi_{\omega} \in \mathcal{G}_{\omega}$ and $\delta>0$, we put

$$
U_{\delta}\left(\phi_{\omega}\right):=\left\{v \in H^{1}\left(\mathbb{R}^{n}\right): \inf _{\theta \in \mathbb{R}}\left\|v-e^{i \theta} \phi_{\omega}\right\|_{H^{1}}<\delta\right\} .
$$

We say that a standing wave solution $e^{i \omega t} \phi_{\omega}(x)$ of $(1.1)$ is stable in $H^{1}\left(\mathbb{R}^{n}\right)$ if for any $\varepsilon>0$ there exists $\delta>0$ such that for any $u_{0} \in U_{\delta}\left(\phi_{\omega}\right)$, the solution $u(t)$ of (1.1) with $u(0)=u_{0}$ satisfies $u(t) \in U_{\varepsilon}\left(\phi_{\omega}\right)$ for any $t \geq 0$. Otherwise, $e^{i \omega t} \phi_{\omega}(x)$ is said to be unstable in $H^{1}\left(\mathbb{R}^{n}\right)$.

The following theorem is our main result in this paper.

Theorem 1 Let $n \geq 3$ and $1<p<1+(4-2 b) / n$. Assume (V1) and (V2). Let $\phi_{\omega} \in \mathcal{G}_{\omega}$. Then, there exists $\omega_{*}>0$ such that $e^{i \omega t} \phi_{\omega}(x)$ is stable in $H^{1}\left(\mathbb{R}^{n}\right)$ for any $\omega \in\left(0, \omega_{*}\right)$. In particular, we can take $\omega_{*}=\infty$ in the case where $V(x)=|x|^{-b}$ with $0<b<2$.

Remark 1.2 We make use of Hardy's type inequality to control the degree of nonlinearity in the space $H^{1}\left(\mathbb{R}^{n}\right)$. That is why the restriction on the spatial dimensions, i.e., $n \geq 3$ appears in the assumption of Theorem 1.

Grillakis, Shatah and Strauss $[13,14]$ gave an almost sufficient and necessary condition for the stability and instability of stationary states for the Hamiltonian systems under certain assumptions. By the abstract theory in Grillakis, Shatah and Strauss [13,14], under some assumptions on the spectrum of linearized operators, $e^{i \omega_{0} t} \phi_{\omega_{0}}(x)$ is stable (resp. unstable) if the function $\left\|\phi_{\omega}\right\|_{2}^{2}$ is strictly increasing (resp. decreasing) at $\omega=\omega_{0}$. In the papers of Shatah [24], Shatah and Strauss [25], the authors used the variational characterization of ground states instead of assumptions on the spectrum of linearized operators. In the case $V(x) \equiv 1$, by the scaling $\psi_{\omega}(x)=\omega^{1 /(p-1)} \psi_{1}(\sqrt{\omega} x)$, it is easy to check the increase and decrease of $\left\|\psi_{\omega}\right\|_{2}^{2}$. However, it seems difficult to check this property of $\left\|\phi_{\omega}\right\|_{2}^{2}$ for $V(x) \not \equiv 1$ since we do not have the scaling invariance in general.

To avoid such difficulty, we apply another sufficient condition for stability.

Proposition 2 Let $n \geq 3$ and $1<p<1+(4-2 b) /(n-2)$. Assume (V1) and $\lim _{|x| \rightarrow \infty} V(x)=0$. Let $\phi_{\omega} \in \mathcal{G}_{\omega}$. If there exists $\delta>0$ such that

$$
\left\langle S_{\omega}^{\prime \prime}\left(\phi_{\omega}\right) v, v\right\rangle \geq \delta\|v\|_{H^{1}}^{2}
$$

for any $v \in H^{1}\left(\mathbb{R}^{n}\right)$ satisfying $\operatorname{Re}\left(\phi_{\omega}, v\right)_{L^{2}}=0$ and $\operatorname{Re}\left(i \phi_{\omega}, v\right)_{L^{2}}=0$, then the standing wave solution $e^{i \omega t} \phi_{\omega}(x)$ of $(1.1)$ is stable in $H^{1}\left(\mathbb{R}^{n}\right)$.

Remark 1.3 In Proposition 2, the condition $\operatorname{Re}\left(\phi_{\omega}, v\right)_{L^{2}}=0$ is related to the conservation of charge $Q$. In fact, we have $\left\langle Q^{\prime}\left(\phi_{\omega}\right), v\right\rangle=\operatorname{Re}\left(\phi_{\omega}, v\right)_{L^{2}}$. Moreover, since it follows from $S_{\omega}^{\prime}\left(e^{i \theta} \phi_{\omega}\right)=0$ for $\theta \in \mathbb{R}$ that $S_{\omega}^{\prime \prime}\left(\phi_{\omega}\right) i \phi_{\omega}=0,(1.6)$ does not hold if we do not restrict $v \in H^{1}\left(\mathbb{R}^{n}\right)$ to satisfy $\operatorname{Re}\left(i \phi_{\omega}, v\right)_{L^{2}}=0$. 
To check this sufficient condition (1.6) for the case of $V(x)$ satisfying (V1) and (V2), we first consider the case where $V(x)=|x|^{-b}$ with $0<b<2$ as a limiting problem since the stability results are already known in the case $V(x)=|x|^{-b}$, which simply follow from the arguments by [24] and [25]. Indeed, in [11], the authors investigated the rescaling limit of $\phi_{\omega}(x)$ as $\omega \rightarrow 0$. It was shown in [11] that as $\omega \rightarrow 0$, the rescaled function $\tilde{\phi}_{\omega}(x)$ defined by

$$
\phi_{\omega}(x)=\omega^{(2-b) / 2(p-1)} \tilde{\phi}_{\omega}(\sqrt{\omega} x), \quad \omega>0
$$

tends to the unique positive radial solution $\psi_{1, b}(x)$ of $(1.2)$ with $\omega=1$ and $V(x)=$ $|x|^{-b}$. Using this convergence, they proved in [11] that $e^{i \omega t} \phi_{\omega}(x)$ is unstable for $p>1+(4-2 b) / n$ and sufficiently small $\omega>0$. Due to the inhomogeneous medium, the standing wave solution tends to be more unstable for small $\omega>0$ since $1+(4-2 b) / n<p<1+4 / n$ is the stability region in the case where $V(x) \equiv 1$.

From known stability properties of $\psi_{1, b}(x)$ (see Section 2 of [11]), we would be able to prove (1.6) in the limit. However, to our knowledge, there is no verification of (1.6) even in the case $V(x)=|x|^{-b}$. For that reason, in Section 2, we first study the properties of the linearized operator at standing wave solution for the case where $V(x)=|x|^{-b}$ in (1.1). In Section 3, we continue analyzing the linearized operator, in particular, we observe that the kernel of real part of the linearized operator is only zero, following the method of Kabeya and Tanaka [18]. We remark that their idea could not be applied directly to our case. We need to modify their perturbed functional in order that the singularity of $|x|^{-b}$ at the origin does not affect the linear part of the equation (1.2). The crucial part is Section 3 because uniqueness and nondegeneracy of a solution of semilinear elliptic equations often plays an essential role in stability problems. In Section 4, we check the condition (1.6) for $V(x)$ satisfying (V1) and (V2), following Esteban and Strauss [8] (see also [10]) and we prove Theorem 1.

We remark that Fibich and Wang [9] and Liu, Wang and Wang [22] treated the stability and instability problems of standing waves for (1.1) with $g\left(x,|u|^{2}\right)=$ $V(\varepsilon x)|u|^{4 / n}$ in a radial space, where $\varepsilon$ is a small parameter. Their ways of proof are also a sort of perturbation method. However, they use (1.4) with $p=1+4 / n$ as a limiting equation, their assumptions for $V(x)$ are different from those in this paper and it is not clear whether there exists a simple relation between $\varepsilon$ and $\omega$.

\section{The case $V(x)=|x|^{-b}$}

We consider the stability of standing waves for

$$
i \partial_{t} u=-\Delta u-\frac{1}{|x|^{b}}|u|^{p-1} u, \quad(t, x) \in \mathbb{R}^{1+n},
$$

where $n \geq 3,0<b<2$ and $1<p<1+(4-2 b) /(n-2)$. 
For any $\omega>0$ there exists a unique positive radial solution $\psi_{\omega, b} \in H^{1}\left(\mathbb{R}^{n}\right)$ of

$$
-\Delta \psi+\omega \psi-\frac{1}{|x|^{b}}|\psi|^{p-1} \psi=0, \quad x \in \mathbb{R}^{n} .
$$

See Stuart [27] and Remark 3.1 of [11] for existence. The positivity of solutions follows from the maximum principle. Radial symmetry of solutions was showed by Gidas, Ni and Nirenberg [12] and Li [20] (see also Li and Ni [21]), and Yanagida [33] proved the uniqueness. Moreover $\psi_{\omega, b}$ is in $C^{2}\left(\mathbb{R}^{n}\right)$ and vanishes as $|x| \rightarrow \infty$, particularly decays exponentially (see $[4,5]$ ). This unique solution is a minimizer of

$$
d_{b}(\omega):=\inf \left\{S_{\omega, b}(v): v \in H^{1}\left(\mathbb{R}^{n}\right) \backslash\{0\}, I_{\omega, b}(v)=0\right\},
$$

where

$$
\begin{gathered}
S_{\omega, b}(v)=\frac{1}{2}\|\nabla v\|_{2}^{2}+\frac{\omega}{2}\|v\|_{2}^{2}-\frac{1}{p+1} \int_{\mathbb{R}^{n}} \frac{1}{|x|^{b}}|v(x)|^{p+1} d x, \\
I_{\omega, b}(v)=\|\nabla v\|_{2}^{2}+\omega\|v\|_{2}^{2}-\int_{\mathbb{R}^{n}} \frac{1}{|x|^{b}}|v(x)|^{p+1} d x .
\end{gathered}
$$

In this section, we note the following fact as a special case of Theorem 1 .

Proposition 3 Let $n \geq 3,0<b<2$ and $1<p<1+(4-2 b) / n$. Then the standing wave solution $e^{i \omega t} \psi_{\omega, b}(x)$ of $(2.1)$ is stable in $H^{1}\left(\mathbb{R}^{n}\right)$ for any $\omega>0$.

Actually, this fact can be proved simply by applying the method of $[24,25]$ to the present case. Using the variational characterization $d_{b}(\omega)$, we may check the sufficient condition for stability $d_{b}^{\prime \prime}(\omega)>0$ in [24] and instability $d_{b}^{\prime \prime}(\omega)<0$ in [25]. Since $\psi_{\omega, b}(x)$ is a solution of $S_{\omega, b}^{\prime}(v)=0$, we have $d_{b}^{\prime}(\omega)=Q\left(\psi_{\omega, b}\right)$. In this case, by the scaling $\psi_{\omega, b}(x)=\omega^{(2-b) / 2(p-1)} \psi_{1, b}(\sqrt{\omega} x)$, we have $2 Q\left(\psi_{\omega, b}\right)=$ $\left\|\psi_{\omega, b}\right\|_{2}^{2}=\omega^{\{(2-b) /(p-1)\}-n / 2}\left\|\psi_{1, b}\right\|_{2}^{2}$. Therefore, for any $\omega>0$, the standing wave solution is stable if $1<p<1+(4-2 b) / n$, and unstable if $1+(4-2 b) / n<p<$ $1+(4-2 b) /(n-2)$. We have also blow-up instability for the case $p \geq 1+(4-2 b) / n$, following Weinstein [30] and Berestycki and Cazenave [3].

However, stability of standing wave solution does not always seem to imply (1.6) immediately. The constraints in (1.6) depend on the negative and zero eigenvalues of the linearized operator at $\psi_{\omega, b}$. Therefore, the main aim in this section is to show the following proposition.

Proposition 4 Assume $n \geq 3,0<b<2$ and $1<p<1+(4-2 b) / n$. Let $\psi_{1, b}(x)$ be the unique positive radial solution of (2.2) with $\omega=1$. Then there exists $\delta>0$ such that

$$
\left\langle S_{1, b}^{\prime \prime}\left(\psi_{1, b}\right) v, v\right\rangle \geq \delta\|v\|_{H^{1}}^{2}
$$

for any $v \in H^{1}\left(\mathbb{R}^{n}\right)$ satisfying $\operatorname{Re}\left(\psi_{1, b}, v\right)_{L^{2}}=0$ and $\operatorname{Re}\left(i \psi_{1, b}, v\right)_{L^{2}}=0$. 
Remark 2.1 By combining this proposition with Proposition 2, it follows that the standing wave solution $e^{i t} \psi_{1, b}(x)$ of $(2.1)$ is stable in $H^{1}\left(\mathbb{R}^{n}\right)$, that is, Proposition 3 holds.

We define two self-adjoint operators $L_{1, b}$ and $L_{2, b}$ on $L^{2}\left(\mathbb{R}^{n}\right)$ by

$$
L_{1, b}=-\Delta+1-p \frac{1}{|x|^{b}} \psi_{1, b}^{p-1}(x), \quad L_{2, b}=-\Delta+1-\frac{1}{|x|^{b}} \psi_{1, b}^{p-1}(x)
$$

with domain $D\left(L_{j, b}\right)=\left\{v \in H^{2}\left(\mathbb{R}^{n}, \mathbb{R}\right):|x|^{-b} \psi_{1, b}^{p-1} v \in L^{2}\left(\mathbb{R}^{n}\right)\right\}$ for $j=1,2$. We remark that for $v \in H^{1}\left(\mathbb{R}^{n}\right)$ with $v_{1}(x)=\operatorname{Re} v(x)$ and $v_{2}(x)=\operatorname{Im} v(x)$,

$$
\begin{aligned}
& \left\langle S_{1, b}^{\prime \prime}\left(\psi_{1, b}\right) v, v\right\rangle=\left\langle L_{1, b} v_{1}, v_{1}\right\rangle+\left\langle L_{2, b} v_{2}, v_{2}\right\rangle, \\
& \left\langle L_{1, b} v_{1}, v_{1}\right\rangle=\left\|v_{1}\right\|_{H^{1}}^{2}-p \int_{\mathbb{R}^{n}} \frac{1}{|x|^{b}} \psi_{1, b}^{p-1}(x)\left|v_{1}(x)\right|^{2} d x, \\
& \left\langle L_{2, b} v_{2}, v_{2}\right\rangle=\left\|v_{2}\right\|_{H^{1}}^{2}-\int_{\mathbb{R}^{n}} \frac{1}{|x|^{b}} \psi_{1, b}^{p-1}(x)\left|v_{2}(x)\right|^{2} d x,
\end{aligned}
$$

and

$$
\operatorname{Re}\left(\psi_{1, b}, v\right)_{L^{2}}=\left(\psi_{1, b}, v_{1}\right)_{L^{2}}, \quad \operatorname{Re}\left(i \psi_{1, b}, v\right)_{L^{2}}=\left(\psi_{1, b}, v_{2}\right)_{L^{2}} .
$$

Thus it suffices to show the following.

Lemma 2.1 Assume $n \geq 3,0<b<2$ and $1<p<1+(4-2 b) /(n-2)$. Let $\psi_{1, b}(x)$ be the unique positive radial solution of (2.2) with $\omega=1$.

(i) If $p<1+(4-2 b) / n$, then there exists $\delta_{1}>0$ such that

$$
\left\langle L_{1, b} v, v\right\rangle \geq \delta_{1}\|v\|_{H^{1}}^{2}
$$

for any $v \in H^{1}\left(\mathbb{R}^{n}, \mathbb{R}\right)$ satisfying $\left(v, \psi_{1, b}\right)_{L^{2}}=0$.

(ii) There exists $\delta_{2}>0$ such that

$$
\left\langle L_{2, b} v, v\right\rangle \geq \delta_{2}\|v\|_{L^{2}}^{2}
$$

for any $v \in H^{1}\left(\mathbb{R}^{n}, \mathbb{R}\right)$ satisfying $\left(v, \psi_{1, b}\right)_{L^{2}}=0$.

The part (ii) of Lemma 2.1 is obtained since $L_{2, b} \psi_{1, b}=0$ and $\psi_{1, b}(x)>0$ for $x \in \mathbb{R}^{n}$. Namely, $\psi_{1, b}$ is the first eigenfunction of $L_{2, b}$ corresponding to the eigenvalue 0 . Moreover, by Weyl's theorem, the essential spectrum of $L_{2, b}$ are in $[1, \infty)$, since $\psi_{1, b}$ tends to zero at infinity. These conclude (ii).

Therefore, we prove the part (i) of Lemma 2.1. For that purpose, we need to show the following two propositions.

Proposition 5 Assume $n \geq 3,0<b<2$ and $1<p<1+(4-2 b) /(n-2)$. If $v \in H^{1}\left(\mathbb{R}^{n}, \mathbb{R}\right)$ satisfies $L_{1, b} v=0$, then $v \equiv 0$. 
Proposition 6 Assume $n \geq 3,0<b<2$ and $1<p \leq 1+(4-2 b) / n$. Then we have

$$
\inf \left\{\left\langle L_{1, b} v, v\right\rangle: v \in H^{1}\left(\mathbb{R}^{n}, \mathbb{R}\right),\left(v, \psi_{1, b}\right)_{L^{2}}=0\right\}=0 .
$$

We shall prove Proposition 5 in the next section. As to Proposition 6, we give a proof in the same way as Proposition 2.7 in Weinstein [31]. First, we show the following lemma.

Lemma 2.2 Assume $n \geq 3,0<b<2$ and $1<p<1+(4-2 b) /(n-2)$. For $v \in H^{1}\left(\mathbb{R}^{n}\right)$, we define the functional

$$
J(v)=\frac{\|\nabla v\|_{2}^{\theta}\|v\|_{2}^{\gamma}}{\int \frac{1}{|x|^{b}}|v|^{p+1}}
$$

where $\theta=\{n(p-1)\} / 2+b>0$ and $\gamma=\{n+2-(n-2) p-2 b\} / 2>0$. Then,

$$
\alpha:=\inf \left\{J(v): v \in H^{1}\left(\mathbb{R}^{n}\right)\right\}
$$

is attained at a positive radial function $\psi^{*}(x) \in H^{1}\left(\mathbb{R}^{n}\right) \cap C^{\infty}\left(\mathbb{R}^{n}\right)$ such that

$$
\psi_{*}(x)=\left(\frac{\gamma^{1-b / 2} \theta^{b / 2}}{\alpha(p+1)}\right)^{1 /(p-1)} \psi_{1, b}\left(\gamma^{1 / 2} \theta^{-1 / 2} x\right) .
$$

Proof. We follow the proof of Theorem B of [30]. Since $J(v) \geq 0$, there exists a minimizing sequence $\left\{v_{\nu}\right\} \subset H^{1}\left(\mathbb{R}^{n}\right)$, that is, $\lim _{\nu \rightarrow \infty} J\left(v_{\nu}\right)=\alpha$. We can assume that $v_{\nu}$ is positive since $\|\nabla|v|\|_{2} \leq\|\nabla v\|_{2}$. Now, let $v^{\lambda, \mu}(x)=\lambda v(\mu x)$ for $\lambda, \mu>0$. Then we have

$$
\begin{aligned}
& J\left(v^{\lambda, \mu}\right)=J(v), \\
& \left\|\nabla v^{\lambda, \mu}\right\|_{2}^{2}=\lambda^{2} \mu^{2-n}\|\nabla v\|_{2}^{2}, \\
& \left\|v^{\lambda, \mu}\right\|_{2}^{2}=\lambda^{2} \mu^{-n}\|v\|_{2}^{2}, \\
& \int \frac{1}{|x|^{b}}\left|v^{\lambda, \mu}\right|^{p+1}=\lambda^{p+1} \mu^{-n+b} \int \frac{1}{|x|^{b}}|v|^{p+1} .
\end{aligned}
$$

We choose $\mu_{\nu}=\left\|v_{\nu}\right\|_{2} /\left\|\nabla v_{\nu}\right\|_{2}$ and $\lambda_{\nu}=\left\|v_{\nu}\right\|_{2}^{n / 2-1} /\left\|\nabla v_{\nu}\right\|_{2}^{n / 2}$ so that $\psi_{\nu}:=$ $v^{\lambda_{\nu}, \mu_{\nu}}$ has the following properties.

$$
\begin{aligned}
& \psi_{\nu}(x) \in H^{1}\left(\mathbb{R}^{n}\right), \quad \psi_{\nu}(x) \geq 0, \quad x \in \mathbb{R}^{n}, \\
& \left\|\psi_{\nu}\right\|_{2}=1, \quad\left\|\nabla \psi_{\nu}\right\|_{2}^{2}=1 \\
& J\left(\psi_{\nu}\right) \rightarrow \alpha \quad \text { as } \quad \nu \rightarrow \infty
\end{aligned}
$$

Namely $\left\{\psi_{\nu}\right\}$ is bounded in $H^{1}\left(\mathbb{R}^{n}\right)$. Thus there exists a subsequence $\left\{\psi_{\nu}\right\}$ and a limit $\psi_{*}(x) \in H^{1}\left(\mathbb{R}^{n}\right)$ such that $\psi_{\nu}$ converges to $\psi_{*}$ weakly in $H^{1}\left(\mathbb{R}^{n}\right)$. It follows 
from the Sobolev embedding on a bounded domain and the smallness of $|x|^{-b}$ for large $|x|$ that

$$
\int_{\mathbb{R}^{n}} \frac{1}{|x|^{b}} \psi_{\nu}^{p+1}(x) d x \rightarrow \int_{\mathbb{R}^{n}} \frac{1}{|x|^{b}} \psi_{*}^{p+1}(x) d x \quad \text { as } \quad \nu \rightarrow \infty
$$

for $1<p<1+(4-2 b) /(n-2)$ (see the argument in [27], Lemma 1.1 and Remark 3.1 of [11]). By weak convergence, $\left\|\psi_{*}\right\|_{2} \leq 1$ and $\left\|\nabla \psi_{*}\right\|_{2} \leq 1$. Furthermore,

$$
\begin{aligned}
\alpha \leq J\left(\psi_{*}\right)=\frac{\left\|\nabla \psi_{*}\right\|_{2}^{\theta}\left\|\psi_{*}\right\|_{2}^{\gamma}}{\int \frac{1}{|x|^{b}}\left|\psi_{*}\right|^{p+1}} & \leq \liminf _{\nu \rightarrow \infty} \frac{\left\|\nabla \psi_{\nu}\right\|_{2}^{\theta}\left\|\psi_{\nu}\right\|_{2}^{\gamma}}{\int \frac{1}{|x|^{b}}\left|\psi_{\nu}\right|^{p+1}}=\liminf _{\nu \rightarrow \infty} J\left(\psi_{\nu}\right) \\
& =\liminf _{\nu \rightarrow \infty} \frac{1}{\int \frac{1}{|x|^{b}}\left|\psi_{\nu}\right|^{p+1}}=\alpha
\end{aligned}
$$

It follows that $\left\|\nabla \psi_{*}\right\|_{2}^{\theta}\left\|\psi_{*}\right\|_{2}^{\gamma}=1$ and therefore $\left\|\nabla \psi_{*}\right\|_{2}=\left\|\psi_{*}\right\|_{2}=1$, which implies that $\psi_{\nu} \rightarrow \psi_{*}$ strongly in $H^{1}\left(\mathbb{R}^{n}\right)$. This minimizing function $\psi_{*}$ satisfies the Euler-Lagrange equation:

$$
\left.\frac{d}{d \varepsilon} J\left(\psi_{*}+\varepsilon \eta\right)\right|_{\varepsilon=0}=0 \quad \text { for any } \eta \in C_{0}^{\infty}\left(\mathbb{R}^{n}\right)
$$

Taking into account that $\left\|\nabla \psi_{*}\right\|_{2}=\left\|\psi_{*}\right\|_{2}=1$ and that $\int|x|^{-b}\left|\psi_{*}\right|^{p+1}=1 / \alpha$, we have

$$
-\theta \Delta \psi_{*}+\gamma \psi_{*}-\alpha(p+1) \frac{1}{|x|^{b}} \psi_{*}^{p}=0
$$

The smoothness of $\psi_{*}$ follows from the same method as Section 8 of Cazenave [5].

The scaling $\psi_{*}(x)=\left(\frac{\gamma^{1-b / 2} \theta^{b / 2}}{\alpha(p+1)}\right)^{1 /(p-1)} \psi\left(\gamma^{1 / 2} \theta^{-1 / 2} x\right)$ makes $\psi(x)$ be a positive solution of (2.2) with $\omega=1$. By the results in [12] and [20], $\psi(x)$ is radial. Accordingly, $\psi(x)$ is the unique solution $\psi_{1, b}(r)$.

Proof of Proposition 6. We remark that the infimum of (2.3) is nonpositive because the value $\left\langle L_{1, b} v, v\right\rangle$ is zero for $v=0$. Since $J(v)$ attains its minimum at $\psi_{1, b}$,

$$
\left.\frac{d^{2}}{d \varepsilon^{2}} J\left(\psi_{1, b}+\varepsilon \eta\right)\right|_{\varepsilon=0} \geq 0
$$

for all $\eta \in C_{0}^{\infty}\left(\mathbb{R}^{n}\right)$. A simple calculation concludes

$$
\left\langle L_{1, b} v, v\right\rangle \geq \frac{2 \theta}{\alpha}\left(1-\frac{\theta}{2}\right)\left(\nabla \psi_{1, b}, \nabla v\right)_{L^{2}}^{2}
$$

for any $v \in H^{1}\left(\mathbb{R}^{n}, \mathbb{R}\right)$ with $\left(v, \psi_{1, b}\right)_{L^{2}}=0$, where $\alpha$ and $\theta$ have been defined in Lemma 2.2. The result follows since the right-hand side of $(2.4)$ is nonnegative for $p \leq 1+(4-2 b) / n$. 
Now we are ready to give a proof of part (i) of Lemma 2.1.

Proof of Lemma 2.1 (i). Let

$$
\tau:=\inf \left\{\left\langle L_{1, b} v, v\right\rangle: v \in H^{1}\left(\mathbb{R}^{n}, \mathbb{R}\right),\left(v, \psi_{1, b}\right)_{L^{2}}=0,\|v\|_{H^{1}}=1\right\}
$$

and suppose $\tau=0$ under the condition $1<p<1+(4-2 b) / n$. Let $\left\{v_{j}\right\} \subset H^{1}\left(\mathbb{R}^{n}\right)$ be a minimizing sequence, that is,

$$
\begin{aligned}
& \lim _{j \rightarrow \infty}\left\langle L_{1, b} v_{j}, v_{j}\right\rangle=0, \\
& \left\|v_{j}\right\|_{H^{1}}=1, \quad\left(v_{j}, \psi_{1, b}\right)_{L^{2}}=0 .
\end{aligned}
$$

Since $\left\{v_{j}\right\}$ is bounded in $H^{1}\left(\mathbb{R}^{n}\right)$, there exists a subsequence still denoted by $\left\{v_{j}\right\} \subset H^{1}\left(\mathbb{R}^{n}, \mathbb{R}\right)$ which converges weakly to some $f_{*} \in H^{1}$. By weak convergence, $f_{*}$ satisfies $\left(f_{*}, \psi_{1, b}\right)_{L^{2}}=0$. We also have

$$
\int \frac{1}{|x|^{b}} \psi_{1, b}^{p-1} v_{j}^{2} \rightarrow \int \frac{1}{|x|^{b}} \psi_{1, b}^{p-1} f_{*}^{2}
$$

as $j \rightarrow \infty$ for $1<p<1+(4-2 b) /(n-2)$. Indeed, we note that $v_{j}^{2}$ converges weakly to $f_{*}^{2}$ in $L^{n /(n-2)}\left(\mathbb{R}^{n}\right)$ by the Sobolev embedding, and that $|x|^{-b} \psi_{1, b}^{p-1}(x) \in$ $L^{n / 2}\left(\mathbb{R}^{n}\right)$ since $|x|^{-b}$ vanishes at infinity and $\psi_{1, b}(x)$ decays exponentially for $|x| \geq$ $C$ with some $C>0$. For $|x| \leq C$, we know that $|x|^{-b} \psi_{1, b}^{p-1}(x) \in L^{n / 2}(|x| \leq C)$ if $p<1+(4-2 b) /(n-2)$. Thus, we have

$$
\begin{aligned}
& 0=\lim _{j \rightarrow \infty}\left\langle L_{1, b} v_{j}, v_{j}\right\rangle \\
& =1-p \lim _{j \rightarrow \infty} \int_{\mathbb{R}^{n}} \frac{1}{|x|^{b}} \psi_{1, b}^{p-1} v_{j}^{2} \\
& =1-p \int_{\mathbb{R}^{n}} \frac{1}{|x|^{b}} \psi_{1, b}^{p-1} f_{*}^{2}
\end{aligned}
$$

and then, $f_{*} \not \equiv 0$. Moreover, by weak convergence, $\left\|f_{*}\right\|_{H^{1}} \leq 1$ and

$$
0 \leq\left\langle L_{1, b} f_{*}, f_{*}\right\rangle \leq \lim _{j \rightarrow \infty}\left\langle L_{1, b} v_{j}, v_{j}\right\rangle=0,
$$

where the first inequality follows from Proposition 6. We define $g_{*}:=f_{*} /\left\|f_{*}\right\|_{H^{1}}$ and then $g_{*}$ satisfies $g_{*} \in H^{1}\left(\mathbb{R}^{n}\right),\left\|g_{*}\right\|_{H^{1}}=1,\left(g_{*}, \psi_{1, b}\right)_{L^{2}}=0, g_{*} \not \equiv 0$ and $\left\langle L_{1, b} g_{*}, g_{*}\right\rangle=0$. Since the minimum is attained at an admissible function $g_{*} \not \equiv 0$, there exists $\left(g_{*}, \lambda, \beta\right)$ solution of the Lagrange multiplier problem

$$
\begin{aligned}
& L_{1, b} g_{*}=\lambda\left(-\Delta g_{*}+g_{*}\right)+\beta \psi_{1, b}, \quad \lambda, \beta \in \mathbb{R}, \\
& \left\|g_{*}\right\|_{H^{1}}=1 \\
& \left(g_{*}, \psi_{1, b}\right)_{L^{2}}=0
\end{aligned}
$$


By (2.6), (2.7) and (2.8), $\lambda=\left\langle L_{1, b} g_{*}, g_{*}\right\rangle$. Thus, $\lambda=0$ since we have assumed $\tau=0$. Therefore,

$$
L_{1, b} g_{*}=\beta \psi_{1, b} \text {. }
$$

On the other hand, let

$$
g:=\frac{b-2}{2}\left(\frac{1}{p-1} \psi_{1, b}+\frac{1}{2-b} x \cdot \nabla \psi_{1, b}\right) .
$$

Then we have $L_{1, b} g=\psi_{1, b}$. Accordingly, $L_{1, b}\left(g_{*}-\beta g\right)=0$. It follows from Proposition 5 that $g_{*}=\beta g$. If $\beta=0$, then $g_{*}=0$, which is a contradiction. Thus $\beta \neq 0$. Here,

$$
\left(g_{*}, \psi_{1, b}\right)_{L^{2}}=\left(\beta g, \psi_{1, b}\right)_{L^{2}}=-\frac{\beta}{2}\left(\frac{2-b}{p-1}-\frac{n}{2}\right)\left\|\psi_{1, b}\right\|_{2}^{2},
$$

which violates $(2.8)$ when $p<1+(4-2 b) / n$. Thus, $g_{*} \equiv 0$, a contradiction. We now conclude that $\tau>0$ if $p<1+(4-2 b) / n$.

\section{Nondegeneracy of unique positive radial solution for (2.2)}

In this section, we give a proof of Proposition 5, following Kabeya and Tanaka [18]. We always assume that $n \geq 3,0<b<2$ and $1<p<1+(4-2 b) /(n-2)$.

Let $\psi_{1, b}(r) \in H^{1}\left(\mathbb{R}^{n}\right)$ be the unique positive radial solution of $(2.2) \cdot \psi_{1, b}(r)$ decays exponentially and can be characterized as a critical point of the $C^{2}$ functional

$$
S_{1, b,+}(v)=\frac{1}{2}\|\nabla v\|_{2}^{2}+\frac{1}{2}\|v\|_{2}^{2}-\frac{1}{p+1} \int_{\mathbb{R}^{n}} \frac{1}{|x|^{b}} v_{+}^{p+1} d x
$$

where $v_{+}=\max \{v, 0\}$.

Remark 3.1 We briefly explain why $S_{1, b,+}(v)$ is $C^{2}$ on $H^{1}\left(\mathbb{R}^{n}\right)$ when $1<p<$ $1+(4-2 b) /(n-2)$. For $v \in H^{1}\left(\mathbb{R}^{n}\right)$, let

$$
N(v)=\frac{1}{p+1} \int_{\mathbb{R}^{n}} \frac{1}{|x|^{b}} v_{+}^{p+1} d x, \quad M(s)=\int_{0}^{s} m(x, \tau) d \tau,
$$

where $m(x, \tau)=|x|^{-b} \tau_{+}^{p}$. For $v, h \in H^{1}\left(\mathbb{R}^{n}\right)$ and $t \in(-1,1) \backslash\{0\}$, we have

$$
\left|\frac{M(v+t h)-M(v)}{t}\right| \leq C|x|^{-b}\left(\left|v_{+}+t h_{+}\right|^{p}+\left|v_{+}\right|^{p}\right)|h|
$$

since the function $y \rightarrow y_{+}^{p+1}$ is a $C^{2}$ function on $\mathbb{R}$ if $p>1$. The right-hand side of (3.1) belongs to $L^{1}\left(\mathbb{R}^{n}\right)$ if $1<p<1+(4-2 b) /(n-2)$. Therefore, by Lebesgue's convergence theorem,

$$
\begin{aligned}
& \lim _{t \rightarrow 0} \frac{N(v+t h)-N(v)}{t}=\int_{\mathbb{R}^{n}} \lim _{t \rightarrow 0} \frac{M(v+t h)-M(v)}{t} d x \\
& =\int_{\mathbb{R}^{n}} \lim _{t \rightarrow 0} \int_{0}^{t} m(x, v+t h) h d t d x=\int_{\mathbb{R}^{n}} \frac{1}{|x|^{b}} v_{+}^{p} h d x .
\end{aligned}
$$


We conclude $N(v) \in C^{1}\left(H^{1}\left(\mathbb{R}^{n}\right), \mathbb{R}\right)$ and $N^{\prime}(v) h=\int_{\mathbb{R}^{n}}|x|^{-b} v_{+}^{p} h d x$, for $v, h \in$ $H^{1}\left(\mathbb{R}^{n}\right)$. $C^{2}$ regularity follows from the same argument.

Any non-zero critical point of $S_{1, b,+}(v)$ is a positive solution by the maximum principle. On the other hand, as we mentioned in Section 2, radial symmetry of a positive solution and the uniqueness of positive radial solutions follow from $[12,20]$ and [33]. Thus it is $\psi_{1, b}(r)$.

For $\delta>0$ small, we consider the following perturbed functional:

$$
S_{\delta}(v)=S_{1, b,+}(v)-\delta\left(\frac{1}{p+1} \int_{\mathbb{R}^{n}} v_{+}^{p+1} d x-\frac{1}{2} \int_{\mathbb{R}^{n}} \psi_{1, b}^{p-1} v^{2} d x\right) .
$$

Critical points $v(x)$ of $S_{\delta}(v)$ satisfy

$$
-\Delta v+\left(1+\delta \psi_{1, b}^{p-1}\right) v=\left(\frac{1}{|x|^{b}}+\delta\right) v_{+}^{p}, \quad x \in \mathbb{R}^{n} .
$$

By the maximum principle, non-zero solutions are positive. Furthermore, such positive solutions are radial for small $\delta>0$ (see $[12,20])$. Thus they satisfy

$$
\begin{aligned}
& -\Delta v+\left(1+\delta \psi_{1, b}^{p-1}\right) v=\left(\frac{1}{|x|^{b}}+\delta\right) v^{p}, \quad x \in \mathbb{R}^{n}, \\
& v(x)>0, \quad v(x)=v(|x|), \quad x \in \mathbb{R}^{n} \\
& v \in H^{1}\left(\mathbb{R}^{n}\right) .
\end{aligned}
$$

By Yanagida [33], we see that (3.2)-(3.4) has a unique positive radial solution for small $\delta>0$ (see Appendix). Since $\psi_{1, b}(r)$ satisfies (3.2)-(3.4), the unique solution of $(3.2)-(3.4)$ is $\psi_{1, b}(r)$.

For $\delta \geq 0$, we define the Morse index

index $S_{\delta}^{\prime \prime}\left(\psi_{1, b}\right)=\max \left\{\operatorname{dim} H: H \subset H^{1}\left(\mathbb{R}^{n}\right)\right.$ is a subspace such that

$$
\left.\left\langle S_{\delta}^{\prime \prime}\left(\psi_{1, b}\right) h, h\right\rangle<0 \text { for all } h \in H \backslash\{0\}\right\} \text {. }
$$

$\psi_{1, b}(r)$ has the following properties.

\section{Lemma 3.1}

(i) For sufficiently small $\delta \geq 0, \psi_{1, b}$ is a mountain pass critical point of $S_{\delta}(v)$, i.e.,

$$
S_{\delta}\left(\psi_{1, b}\right)=\inf _{\gamma \in \Gamma} \max _{s \in[0,1]} S_{\delta}(\gamma(s))
$$

where $\Gamma=\left\{\gamma(s) \in C\left([0,1], H^{1}\left(\mathbb{R}^{n}\right)\right): \gamma(0)=0, \gamma(1)=e_{0}\right\}$. Here, $e_{0} \in$ $H^{1}\left(\mathbb{R}^{n}\right)$ satisfies $S_{\delta}\left(e_{0}\right)<0$.

(ii) The Morse index at $\psi_{1, b}$ is equal to 1 for small $\delta \geq 0$, i.e.,

$$
\text { index } S_{\delta}^{\prime \prime}\left(\psi_{1, b}\right)=1 \text {. }
$$


For the proof of Lemma 3.1, we recall Hofer's result in [15] (see also Tanaka [28] as a related reference).

Proposition 7 ([15]) Let $F$ be a real Hilbert space and $U \subset F$ be a nonempty open subset. Assume that $I \in C^{2}(U, \mathbb{R})$ satisfies Palais-Smale condition and the gradient $I^{\prime}$ has the form identity-K, where $K$ is compact. Define $A, c, d$ by

$$
\begin{aligned}
& A=\left\{a \in C([0,1], F): a(i)=e_{i}, i=0,1\right\} \\
& d=\inf _{a \in A} \sup I(a[0,1]), \\
& c=\max \left\{I\left(e_{0}\right), I\left(e_{1}\right)\right\}
\end{aligned}
$$

and assume $d>c$. Let $u_{0} \in U$ is an isolated critical point of $I$ at the level $d$. Then the Morse index at $u_{0}$ is at most 1.

Proof of Lemma 3.1. (i) For some $\rho_{0}>0$ and $e_{0} \in H^{1}\left(\mathbb{R}^{n}\right)$, we have

$$
\begin{aligned}
& \inf _{\|v\|_{H^{1}}=\rho_{0}} S_{\delta}(v)>0 \\
& \left\|e_{0}\right\|_{H^{1}} \geq \rho_{0} \quad \text { and } \quad S_{\delta}\left(e_{0}\right)<0 .
\end{aligned}
$$

Therefore $S_{\delta}(v)$ has mountain pass geometry. Since the embedding $H^{1} \subset L^{2}$ is compact on a bounded domain and $|x|^{-b}$ vanishes at infinity, $S_{\delta}(v)$ satisfies the Palais-Smale compactness condition if $p<1+(4-2 b) /(n-2)$ (see Lemma 1.1 and Remark 3.1 of [11]) and small $\delta \geq 0$. Therefore we can apply the mountain pass theorem. Since $\psi_{1, b}$ is the unique non-zero critical point of $S_{\delta}(v)$ for sufficiently small $\delta \geq 0, \psi_{1, b}$ is the mountain pass critical point.

(ii) By Proposition 7, the Morse index is at most one at the mountain pass critical point, i.e., index $S_{\delta}^{\prime \prime}\left(\psi_{1, b}\right) \leq 1$. Indeed, $S_{\delta}(v)$ satisfies the conditions in Proposition 7. For $v, h \in H^{1}\left(\mathbb{R}^{n}\right)$, let $S_{\delta}^{\prime}(v) h=\langle v-K(v), h\rangle_{H^{1}}$, where $K(v)=$ $K_{1}(v)+K_{2}(v): H^{1}\left(\mathbb{R}^{n}\right) \rightarrow H^{1}\left(\mathbb{R}^{n}\right)$ defined by $\left\langle K_{1}(v), h\right\rangle_{H^{1}}=\int_{\mathbb{R}^{n}} \delta \psi_{1, b}^{p-1} h d x$, $\left\langle K_{2}(v), h\right\rangle_{H^{1}}=\int_{\mathbb{R}^{n}}\left(|x|^{-b}+\delta\right) v_{+}^{p} h d x$. We see that $K_{1}$ is compact and that $K_{2}$ is compact for sufficiently small $\delta \geq 0$. Furthermore, $\psi_{1, b}$ is the unique mountain pass critical point for sufficiently small $\delta \geq 0$.

On the other hand,

$$
\left\langle S_{\delta}^{\prime \prime}\left(\psi_{1, b}\right) h, h\right\rangle=\|\nabla h\|_{2}^{2}+\int_{\mathbb{R}^{n}}\left(1+\delta \psi_{1, b}^{p-1}\right)|h|^{2}-\int_{\mathbb{R}^{n}} p\left(\frac{1}{|x|^{b}}+\delta\right) \psi_{1, b}^{p-1} h^{2} d x .
$$

Setting $h=\psi_{1, b}$ and using $\left\langle S_{\delta}^{\prime}\left(\psi_{1, b}\right), \psi_{1, b}\right\rangle=0$, we have

$$
\left\langle S_{\delta}^{\prime \prime}\left(\psi_{1, b}\right) \psi_{1, b}, \psi_{1, b}\right\rangle=-(p-1) \int_{\mathbb{R}^{n}}\left(\frac{1}{|x|^{b}}+\delta\right) \psi_{1, b}^{p+1} d x<0
$$

Thus we get index $S_{\delta}^{\prime \prime}\left(\psi_{1, b}\right)=1$. 
Using Lemma 3.1, we verify Proposition 5.

Proof of Proposition 5. Suppose that there exists a non-zero solution $w_{0} \in H^{1}\left(\mathbb{R}^{n}\right)$ of $L_{1, b} w_{0}=0$. It satisfies

$$
\left\langle S_{1, b,+}^{\prime \prime}\left(\psi_{1, b}\right) w_{0}, \xi\right\rangle=0 \quad \text { for all } \xi \in H^{1}\left(\mathbb{R}^{n}\right) .
$$

By Lemma 3.1 (ii) with $\delta=0$, we may also find a $w_{1} \in H^{1}\left(\mathbb{R}^{n}\right)$ such that

$$
\left\langle S_{1, b,+}^{\prime \prime}\left(\psi_{1, b}\right) w_{1}, w_{1}\right\rangle<0 .
$$

We define a 2 -dimensional subspace $H$ of $H^{1}\left(\mathbb{R}^{n}\right)$ by $H=\operatorname{span}\left\{w_{0}, w_{1}\right\}$. Then we have

$$
\left\langle S_{1, b,+}^{\prime \prime}\left(\psi_{1, b}\right) h, h\right\rangle \leq 0 \quad \text { for all } h \in H
$$

On the other hand, we have for all $\delta>0$,

$$
\begin{aligned}
\left\langle S_{\delta}^{\prime \prime}\left(\psi_{1, b}\right) h, h\right\rangle & =\left\langle S_{1, b,+}^{\prime \prime}\left(\psi_{1, b}\right) h, h\right\rangle-\delta(p-1) \int_{\mathbb{R}^{n}} \psi_{1, b}^{p-1} h^{2} d x \\
& \leq-\delta(p-1) \int_{\mathbb{R}^{n}} \psi_{1, b}^{p-1} h^{2} d x \quad \text { for all } h \in H .
\end{aligned}
$$

We remark that $\psi_{1, b}(x)>0$ in $\mathbb{R}^{n}$ and we get

$$
\left\langle S_{\delta}^{\prime \prime}\left(\psi_{1, b}\right) h, h\right\rangle<0 \quad \text { for all } h \in H \backslash\{0\} .
$$

It means that for all $\delta>0$,

$$
\text { index } S_{\delta}^{\prime \prime}\left(\psi_{1, b}\right) \geq 2,
$$

which is a contradiction to Lemma 3.1 (ii) with sufficiently small $\delta \geq 0$.

\section{Proof of Theorem 1}

In this section, we prove the following Lemma 4.1 to show Theorem 1 . For $\omega>0$, we define

$$
\begin{aligned}
& (v, w)_{H^{1}(\omega)}=\operatorname{Re}(\nabla v, \nabla w)_{L^{2}}+\omega \operatorname{Re}(v, w)_{L^{2}}, \\
& \|v\|_{H^{1}(\omega)}=(v, v)_{H^{1}(\omega)}^{1 / 2}, \quad v, w \in H^{1}\left(\mathbb{R}^{n}\right) .
\end{aligned}
$$

Then, we see that $\|\cdot\|_{H^{1}(\omega)}$ is an equivalent norm on $H^{1}\left(\mathbb{R}^{n}\right)$ to $\|\cdot\|_{H^{1}}$.

We remark that for $v \in H^{1}\left(\mathbb{R}^{n}\right)$ with $v_{1}(x)=\operatorname{Re} v(x)$ and $v_{2}(x)=\operatorname{Im} v(x)$, we have

$$
\begin{aligned}
& \left\langle S_{\omega}^{\prime \prime}\left(\phi_{\omega}\right) v, v\right\rangle=\left\langle L_{1, \omega} v_{1}, v_{1}\right\rangle+\left\langle L_{2, \omega} v_{2}, v_{2}\right\rangle \\
& \left\langle L_{1, \omega} v_{1}, v_{1}\right\rangle=\left\|v_{1}\right\|_{H^{1}(\omega)}^{2}-p \int_{\mathbb{R}^{n}} V(x) \phi_{\omega}^{p-1}(x)\left|v_{1}(x)\right|^{2} d x, \\
& \left\langle L_{2, \omega} v_{2}, v_{2}\right\rangle=\left\|v_{2}\right\|_{H^{1}(\omega)}^{2}-\int_{\mathbb{R}^{n}} V(x) \phi_{\omega}^{p-1}(x)\left|v_{2}(x)\right|^{2} d x, \\
& \operatorname{Re}\left(\phi_{\omega}, v\right)_{L^{2}}=\left(\phi_{\omega}, v_{1}\right)_{L^{2}}, \quad \operatorname{Re}\left(i \phi_{\omega}, v\right)_{L^{2}}=\left(\phi_{\omega}, v_{2}\right)_{L^{2}},
\end{aligned}
$$

under the assumptions in Proposition 2. 
Lemma 4.1 Let $n \geq 3,0<b<2$ and $1<p<1+(4-2 b) /(n-2)$. Assume $(\mathrm{V} 1)$ and (V2). Let $\phi_{\omega} \in \mathcal{G}_{\omega}$.

(i) Let $p<1+(4-2 b) / n$. There exists $\omega_{1}>0$ with the following property: for any $\omega \in\left(0, \omega_{1}\right)$, there exists $\delta_{1}>0$ such that

$$
\left\langle L_{1, \omega} v, v\right\rangle \geq \delta_{1}\|v\|_{H^{1}(\omega)}^{2}
$$

for any $v \in H^{1}\left(\mathbb{R}^{n}, \mathbb{R}\right)$ satisfying $\left(v, \phi_{\omega}\right)_{L^{2}}=0$.

(ii) For any $\omega \in(0, \infty)$, there exists $\delta_{2}>0$ such that

$$
\left\langle L_{2, \omega} v, v\right\rangle \geq \delta_{2}\|v\|_{H^{1}(\omega)}^{2}
$$

for any $v \in H^{1}\left(\mathbb{R}^{n}, \mathbb{R}\right)$ satisfying $\left(v, \phi_{\omega}\right)_{L^{2}}=0$.

Proof of Theorem 1. Since $\|\cdot\|_{H^{1}(\omega)}$ is equivalent to $\|\cdot\|_{H^{1}}$, by (4.2) and Lemma 4.1, there exists $\delta>0$ such that (1.6) holds for any $v \in H^{1}\left(\mathbb{R}^{n}\right)$ satisfying $\operatorname{Re}\left(\phi_{\omega}, v\right)_{L^{2}}=0$ and $\operatorname{Re}\left(i \phi_{\omega}, v\right)_{L^{2}}=0$. Hence, Theorem 1 follows from Proposition 2 .

In order to show Lemma 4.1, we use the rescaled function $\tilde{\phi}_{\omega}$ defined by (1.7). For $\omega>0$, we define the rescaled operators $\tilde{L}_{1, \omega}$ and $\tilde{L}_{2, \omega}$ by

$$
\begin{aligned}
& \left\langle\tilde{L}_{1, \omega} v, v\right\rangle=\|v\|_{H^{1}}^{2}-p \omega^{-b / 2} \int_{\mathbb{R}^{n}} V\left(\frac{x}{\sqrt{\omega}}\right) \tilde{\phi}_{\omega}^{p-1}(x)|v(x)|^{2} d x, \\
& \left\langle\tilde{L}_{2, \omega} v, v\right\rangle=\|v\|_{H^{1}}^{2}-\omega^{-b / 2} \int_{\mathbb{R}^{n}} V\left(\frac{x}{\sqrt{\omega}}\right) \tilde{\phi}_{\omega}^{p-1}(x)|v(x)|^{2} d x .
\end{aligned}
$$

Then, for $v(x)=\omega^{(2-b) / 2(p-1)} \tilde{v}(\sqrt{\omega} x)$, we have

$$
\begin{aligned}
\|v\|_{H^{1}(\omega)}^{2} & =\omega^{1+(2-b) /(p-1)-n / 2}\|\tilde{v}\|_{H^{1}}^{2} \\
\left(\phi_{\omega}, v\right)_{L^{2}} & =\omega^{(2-b) /(p-1)-n / 2}\left(\tilde{\phi_{\omega}}, \tilde{v}\right)_{L^{2}} \\
\left\langle L_{j, \omega} v, v\right\rangle & =\omega^{1+(2-b) /(p-1)-n / 2}\left\langle\tilde{L}_{j, \omega} \tilde{v}, \tilde{v}\right\rangle, \quad j=1,2
\end{aligned}
$$

(see (4.1), (4.3) and (4.4)).

Proof of Lemma 4.1. We show (i) by contradiction. Suppose that (i) were false. Then, there would exist $\left\{\omega_{j}\right\}$ and $\left\{v_{j}\right\} \subset H^{1}\left(\mathbb{R}^{n}, \mathbb{R}\right)$ such that $\omega_{j} \rightarrow 0$,

$$
\begin{aligned}
& \lim _{j \rightarrow \infty}\left\langle\tilde{L}_{1, \omega_{j}} v_{j}, v_{j}\right\rangle \leq 0, \\
& \left\|v_{j}\right\|_{H^{1}}^{2}=1, \quad\left(v_{j}, \tilde{\phi}_{\omega_{j}}\right)_{L^{2}}=0 .
\end{aligned}
$$

Since $\left\{v_{j}\right\}$ is bounded in $H^{1}\left(\mathbb{R}^{n}\right)$, there exists a subsequence of $\left\{v_{j}\right\}$ (still denoted by $\left.\left\{v_{j}\right\}\right)$ and $v_{0} \in H^{1}\left(\mathbb{R}^{n}, \mathbb{R}\right)$ such that $v_{j} \rightarrow v_{0}$ weakly in $H^{1}\left(\mathbb{R}^{n}, \mathbb{R}\right)$. Therefore, $\left|v_{j}\right|^{2} \rightarrow\left|v_{0}\right|^{2}$ weakly in $L^{n /(n-2)}\left(\mathbb{R}^{n}\right)$. Further, by Proposition 3 of 
[11], we see that $\tilde{\phi}_{\omega_{j}} \rightarrow \psi_{1}$ strongly in $H^{1}\left(\mathbb{R}^{n}\right)$, so that $\tilde{\phi}_{\omega_{j}}^{p-1} \rightarrow \psi_{1}^{p-1}$ strongly in $L^{2 n /\{(n-2)(p-1)\}}\left(\mathbb{R}^{n}\right) \cap L^{(p+1) /(p-1)}\left(\mathbb{R}^{n}\right)$. Moreover, by (V1) and (V2) if $p<$ $1+(4-2 b) /(n-2)$,

$$
\lim _{j \rightarrow \infty}\left\|\omega_{j}{ }^{-b / 2} V\left(\frac{x}{\sqrt{\omega_{j}}}\right)-\frac{1}{|x|^{b}}\right\|_{\theta^{*}}=0
$$

follows from Lemma 4.2 of [11]. Thus, we have

$$
\lim _{j \rightarrow \infty} \omega_{j}^{-b / 2} \int_{\mathbb{R}^{n}} V\left(\frac{x}{\sqrt{\omega_{j}}}\right) \tilde{\phi}_{\omega_{j}}^{p-1}(x)\left|v_{j}(x)\right|^{2} d x=\int_{\mathbb{R}^{n}} \frac{1}{|x|^{b}} \psi_{1, b}^{p-1}(x)\left|v_{0}(x)\right|^{2} d x .
$$

Indeed,

$$
\begin{aligned}
\int_{\mathbb{R}^{n}}\left(\omega_{j}^{-b / 2}\right. & \left.V\left(\frac{x}{\sqrt{\omega_{j}}}\right) \tilde{\phi}_{\omega_{j}}^{p-1} v_{j}^{2}-\frac{1}{|x|^{b}} \psi_{1, b}^{p-1} v_{0}^{2}\right) d x \\
& =\int_{\mathbb{R}^{n}} \frac{1}{|x|^{b}} \psi_{1, b}^{p-1}\left(v_{j}^{2}-v_{0}^{2}\right) d x+\int_{\mathbb{R}^{n}} \frac{1}{|x|^{b}}\left(\tilde{\phi}_{\omega_{j}}^{p-1}-\psi_{1, b}^{p-1}\right) v_{j}^{2} d x \\
& +\int_{\mathbb{R}^{n}}\left(\omega_{j}^{-b / 2} V\left(\frac{x}{\sqrt{\omega_{j}}}\right)-\frac{1}{|x|^{b}}\right) \tilde{\phi}_{\omega_{j}}^{p-1} v_{j}^{2} d x .
\end{aligned}
$$

The first term converges to 0 as $j \rightarrow \infty$ since $|x|^{-b} \psi_{1, b}^{p-1} \in L^{n / 2}\left(\mathbb{R}^{n}\right)$ (see Proof of Lemma 2.1 (i)). The two remaining terms are estimated as follows: For some $R>0$ such that $|x|^{-b} \leq \varepsilon$ if $|x| \geq R$,

$$
\begin{aligned}
& \int_{\mathbb{R}^{n}} \frac{1}{|x|^{b}}\left(\tilde{\phi}_{\omega_{j}}^{p-1}-\psi_{1, b}^{p-1}\right) v_{j}^{2} d x \\
& \quad \leq\left\||x|^{-b}\right\|_{L^{\theta^{*}}(|x| \leq R)}\left\|\tilde{\phi}_{\omega_{j}}^{p-1}-\psi_{1, b}^{p-1}\right\|_{2 n /\{(n-2)(p-1)\}} \\
& \quad\left\|v_{j}\right\|_{2 n /(n-2)}^{2}+\varepsilon\left\|\tilde{\phi}_{\omega_{j}}^{p-1}-\psi_{1, b}^{p-1}\right\|_{(p+1) /(p-1)}\left\|v_{j}\right\|_{p+1}^{2}, \\
& \int_{\mathbb{R}^{n}}\left(\omega_{j}^{-b / 2} V\left(\frac{x}{\sqrt{\omega_{j}}}\right)-\frac{1}{|x|^{b}}\right) \tilde{\phi}_{\omega_{j}}^{p-1} v_{j}^{2} d x \\
& \quad \leq\left\|\omega_{j}^{-b / 2} V\left(\frac{x}{\sqrt{\omega_{j}}}\right)-\frac{1}{|x|^{b}}\right\|_{\theta^{*}}\left\|\tilde{\phi}_{\omega_{j}}\right\|_{2 n /(n-2)}^{p-1}\left\|v_{j}\right\|_{2 n /(n-2)}^{2},
\end{aligned}
$$

which conclude (4.8). Therefore, by (4.6), (4.7) and (4.8), we have

$$
\begin{aligned}
0 & \geq \liminf _{j \rightarrow \infty}\left\langle\tilde{L}_{1, \omega_{j}} v_{j}, v_{j}\right\rangle \\
& =\liminf _{j \rightarrow \infty}\left(\left\|v_{j}\right\|_{H^{1}}^{2}-p \omega_{j}^{-b / 2} \int_{\mathbb{R}^{n}} V\left(\frac{x}{\sqrt{\omega_{j}}}\right) \tilde{\phi}_{\omega_{j}}^{p-1}(x)\left|v_{j}(x)\right|^{2} d x\right) \\
& =1-p \int_{\mathbb{R}^{n}} \frac{1}{|x|^{b}} \psi_{1, b}^{p-1}(x)\left|v_{0}(x)\right|^{2} d x .
\end{aligned}
$$


Again, by (4.6), (4.8), we have

$$
\begin{aligned}
0 & \geq \liminf _{j \rightarrow \infty}\left\langle\tilde{L}_{1, \omega_{j}} v_{j}, v_{j}\right\rangle \\
& =\liminf _{j \rightarrow \infty}\left(\left\|v_{j}\right\|_{H^{1}}^{2}-p \omega_{j}^{-b / 2} \int_{\mathbb{R}^{n}} V\left(\frac{x}{\sqrt{\omega_{j}}}\right) \tilde{\phi}_{\omega_{j}}^{p-1}(x)\left|v_{j}(x)\right|^{2} d x\right) \\
& \geq\left\|v_{0}\right\|_{H^{1}}^{2}-p \int_{\mathbb{R}^{n}} \frac{1}{|x|^{b}} \psi_{1, b}^{p-1}(x)\left|v_{0}(x)\right|^{2} d x=\left\langle L_{1, b} v_{0}, v_{0}\right\rangle .
\end{aligned}
$$

Moreover, by (4.7), we have $\left(v_{0}, \psi_{1, b}\right)_{L^{2}}=0$. Therefore, by Lemma 2.1 (i), we have $v_{0} \equiv 0$. However, this contradicts (4.9). Hence, we conclude (i). By an analogous argument as (ii) of Lemma 2.1, we can also prove (ii).

\section{Appendix}

\subsection{Uniqueness for (3.2)-(3.4)}

We have cited the uniqueness result by Yanagida [33]. Here, we briefly check the conditions to prove the uniqueness of a solution (3.2)-(3.4). The condition appeared as $(\mathrm{C} 1)-(\mathrm{C} 6)$ in Theorem 2.2 of [33]. In the paper [33], the following type of semilinear elliptic equations was treated:

$$
u^{\prime \prime}(r)+\frac{n-1}{r} u^{\prime}(r)+g(r) u(r)+h(r) u(r)^{p}=0, \quad r>0, \quad n \geq 3,
$$

where we denote $d / d r$ by '.

As an application to our present case, we consider $g(r)=-\left(1+\delta \psi_{1, b}^{p-1}\right)$ and $h(r)=r^{-b}+\delta$, where $\delta \geq 0, n \geq 3,0<b<2$ and $\psi_{1, b}(r)$ is the unique positive radial solution of $(2.2)$ with $\omega=1$. We remark that $\psi_{1, b}(r) \in C^{2}\left(\mathbb{R}^{n}\right)$ decays exponentially as $r \rightarrow \infty$ by the standard argument for radial solutions of elliptic equations (see, for example, Berestycki and Lions [4]) and $\psi_{1, b}(r)$ is monotone decreasing with respect to $r>0$ from $[12,20,21]$, i.e., $\psi_{1, b}^{\prime}(r)<0$ for $r>0$. First, we know that two conditions

(A1) $g(r)$ and $h(r)$ are in $C^{1}((0, \infty))$,

$(\mathrm{A} 2) \quad r^{2-\sigma} g(r) \rightarrow 0$ and $r^{2-\sigma} h(r) \rightarrow 0$ as $r \rightarrow+0$ for some $\sigma>0$,

are satisfied. Now let $m \in[0, n-2]$ be a parameter and define

$$
\begin{aligned}
G(r ; m):= & -\delta(p-1) r^{m+2} \psi_{1, b}^{p-2}(r) \psi_{1, b}^{\prime}(r)+2(n-3-m) r^{m+1}\left(1+\delta \psi_{1, b}^{p-1}(r)\right) \\
& +m(n-2-m)(n-2-m / 2) r^{m-1}, \\
H(r ; m):=\quad & -\left\{2(n-2)-m+\frac{2 b-2(m+2)}{p+1}\right\} r^{m-b+1} \\
& -\left\{2(n-2)-m-\frac{2(m+2)}{p+1}\right\} \delta r^{m+1} .
\end{aligned}
$$

These are related to Pohozaev identity (see Yanagida [33] for details). 
Required conditions in [33, Theorem 2.2] are following:

(C1) $h(r) \geq 0$ for all $r \in(0, \infty)$ and $h(r)>0$ for some $r \in(0, \infty)$.

(C2) $G(r ; n-2) \leq 0$ for all $r \in(0, \infty)$.

(C3) For each $m \in[0, n-2)$, there exists an $\alpha(m) \in[0, \infty]$ such that $G(r ; m) \geq 0$ for $r \in(0, \alpha(m))$ and $G(r ; m) \leq 0$ for $r \in(\alpha(m), \infty)$.

(C4) $H(r ; 0) \leq 0$ for all $r \in(0, \infty)$.

(C5) For each $m \in(0, n-2]$, there exists a $\beta(m) \in[0, \infty]$ such that $H(r ; m) \geq 0$ for $r \in(0, \beta(m))$ and $H(r ; m) \leq 0$ for $r \in(\beta(m), \infty)$.

(C6) When $g(r) \equiv 0$ for all $r \geq 0, h(r)$ satisfies $h(r) \not \equiv C_{0} r^{q}$, where $C_{0}>0$ is an arbitrary constant and $q:=\frac{n-2}{2}\left(p-\frac{n+2}{n-2}\right)$.

The condition (C6) is excluded in the present case. It is clear that (C1), (C4) and (C5) hold since

$$
H(r ; 0)=-\frac{2 b}{p+1} r^{-b+1}-2\left\{n-2-\frac{2}{p+1}\right\} r\left(r^{-b}+\delta\right) .
$$

Also, since

$$
G(r ; n-2)=\left\{r^{2} g(r)\right\}^{\prime}=-r\left(2+2 \delta \psi_{1, b}^{p-1}(r)+\delta r(p-1) \psi_{1, b}^{p-2}(r) \psi_{1, b}^{\prime}(r)\right),
$$

taking $\delta$ so small that the right-hand side is nonpositive for all $r \geq 0$, we can conclude (C2) for sufficiently small $\delta \geq 0$. The condition (C3) follows for small $\delta \geq 0$, too. Indeed, if $0 \leq n-3-m$, then we have $G(r, m)>0$ for all $r>0$, therefore we may take $\alpha(m)=\infty$. If $-1<n-3-m<0$ and $m \geq 1$, we have that $G(r, m) \rightarrow-\infty$ as $r \rightarrow \infty$ and that $G(r, m)$ tends to a nonnegative constant as $r \rightarrow 0$. For the case where $-1<n-3-m<0$ and $m<1$, we see that $G(r, m) \rightarrow-\infty$ as $r \rightarrow \infty$ and $G(r, m) \rightarrow \infty$ as $r \rightarrow 0$. Moreover, in both cases, $\frac{d}{d r}\left(\frac{G^{\prime}(r, m)}{r^{m-2}}\right)<0$ for $r>0$ and sufficiently small $\delta \geq 0$. Thus, there exists $\alpha(m)$ satisfying (C3) (see a similar investigation in [18, Lemma 1.3]).

\subsection{Orbital stability}

Next, we remark on the proof of Proposition 2. Proposition 2 implies the following lemma:

Lemma 5.1 Under the assumptions in Proposition 2, there exist $C>0$ and $\varepsilon>0$ such that

$$
E(u)-E\left(\phi_{\omega}\right) \geq C \inf _{\theta \in \mathbb{R}}\left\|u-e^{i \theta} \phi_{\omega}\right\|_{H^{1}}^{2}
$$

for $u \in U_{\varepsilon}\left(\phi_{\omega}\right)$ with $Q(u)=Q\left(\phi_{\omega}\right)$.

We can prove this lemma following Grillakis, Shatah and Strauss [13, Theorem 3.4] (see also [16, Proposition 1], Section 2 of [10]). Theorem 1 follows from Lemma 5.1 and the proof of Theorem 3.5 of [13]. 


\section{Acknowledgment}

This study started while one of us (R. F) stayed in Université de Paris-Sud, Orsay. R. F is grateful to the staff of Laboratoire d'Analyse Numérique for their warm hospitality. Also, the authors wish to express their sincere appreciation to Professor Kazunaga Tanaka for his helpful advice about Section 3.

\section{References}

[1] N.N. Akhmediev, Novel class of nonlinear surfaces waves: asymmetric modes in a symmetric layered structure, Sov. Phys. JETP 56, 299-303 (1982).

[2] L. Bergé, Soliton stability versus collapse, Phys. Rev. E. 62, R3071-R3074 (2000).

[3] H. Berestycki and T. Cazenave, Instabilité des états stationnaires dans les équations de Schrödinger et de Klein-Gordon non linéaires, C. R. Acad. Sci. Paris. 293, 489-492 (1981).

[4] H. Berestycki and P.L. Lions, Nonlinear scalar field equations, I-Existence of a ground state, Arch. Ration. Mech. Anal. 82, 313-346 (1983).

[5] T. Cazenave, Semilinear Schrödinger equations, Courant Lecture Notes in Mathematics 10, New York University, New York, 2003.

[6] T. Cazenave and P.L. Lions, Orbital stability of standing waves for some nonlinear Schrödinger equations, Comm. Math. Phys. 85, 549-561 (1982).

[7] A. Comech and D. Pelinovsky, Purely nonlinear instability of standing waves with minimal energy, Comm. Pure Appl. Math. 56, 1565-1607 (2003).

[8] M. Esteban and W. Strauss, Nonlinear bound states outside an insulated sphere, Comm. Partial Differential Equations 19, 177-197 (1994).

[9] G. Fibich and X.P. Wang, Stability of solitary waves for nonlinear Schrödinger equations with inhomogeneous nonlinearities, Physica D. 175, 96-108 (2003).

[10] R. Fukuizumi and M. Ohta, Stability of standing waves for nonlinear Schrödinger equations with potentials, Differential and Integral Equations 16, 111-128 (2003).

[11] R. Fukuizumi and M. Ohta, Instability of standing waves for nonlinear Schrödinger equations with inhomogeneous nonlinearities, Preprint.

[12] B. Gidas, W-N. Ni and L. Nirenberg, Symmetry of positive solutions of nonlinear elliptic equations in $\mathbb{R}^{n}$, Math. Anal. and Applications, Part A, Advances in Math. Suppl. Studies 7A, 369-402 (1981). 
[13] M. Grillakis, J. Shatah and W. Strauss, Stability theory of solitary waves in the presence of symmetry I, J. Funct. Anal. 74, 160-197 (1987).

[14] M. Grillakis, J. Shatah and W. Strauss, Stability theory of solitary waves in the presence of symmetry II, J. Funct. Anal. 94, 308-348 (1990).

[15] H. Hofer, A note on the topological degree at a critical point of mountain pass type, Proc. A.M.S. 90, 309-315 (1984).

[16] I.D. Iliev and K.P. Kirchev, Stability and instability of solitary waves for onedimensional singular Schrödinger equations, Differential and Integral Equations 6, 685-703 (1993).

[17] C.K.R.T. Jones, Instability of standing waves for non-linear Schrödinger-type equations, Ergodic Theory Dynam. Systems 8*, 119-138 (1988).

[18] Y. Kabeya and K. Tanaka, Uniqueness of positive radial solutions of semilinear elliptic equations in $\mathbb{R}^{N}$ and Séré's non-degeneracy condition, Comm. Partial Differential Equations 24, 563-598 (1999).

[19] M.K. Kwong, Uniqueness of positive solutions of $\Delta u-u-u^{p}=0$ in $\mathbb{R}^{n}$, Arch. Ration. Mech. Anal. 105, 234-266 (1989).

[20] C. Li, Monotonicity and symmetry of solutions of fully nonlinear elliptic equations on unbounded domains, Comm. Partial Differential Equations 16, 585-615 (1991).

[21] Y. Li and W.-M. Ni, Radial symmetry of positive solutions of nonlinear elliptic equations in $\mathbb{R}^{n}$, Comm. Partial Differential Equations 18, 1043-1054 (1991).

[22] Y. Liu, X.-P. Wang and K. Wang, Instability of standing waves of the Schrödinger equation with inhomogeneous nonlinearity, Preprint.

[23] F. Merle, Nonexistence of minimal blow-up solutions of equations $i u_{t}=$ $-\Delta u-k(x)|u|^{4 / N} u$ in $\mathbb{R}^{n}$, Ann. Inst. H. Poincaré Phys. Théor. 64, 33-85 (1996).

[24] J. Shatah, Stable standing waves for nonlinear Klein-Gordon equations, Comm. Math. Phys. 91, 313-327 (1983).

[25] J. Shatah and W. Strauss, Instability of nonlinear bound states, Comm. Math. Phys. 100, 173-190 (1985).

[26] W. Strauss, Existence of solitary waves in higher dimensions, Comm. Math. Phys. 55, 149-162 (1977).

[27] C.A. Stuart, Bifurcation for Dirichlet problems without eigenvalues, Proc. London Math. Soc. 45, 169-192 (1982). 
[28] K. Tanaka, Morse indices at critical points related to the symmetric mountain pass theorem and applications, Comm. Partial Differential Equations 14, 99128 (1989).

[29] I. Towers and B.A. Malomed, Stable $(2+1)$-dimensional solutions in a layered medium with sign-alternating Kerr nonlinearity, J. Opt. Soc. Am. B 19, 537-543 (2002).

[30] M.I. Weinstein, Nonlinear Schrödinger equations and sharp interpolation estimates, Comm. Math. Phys. 87, 567-576 (1983).

[31] M.I. Weinstein, Modulational stability of ground states of nonlinear Schrödinger equations, Siam J. Math. Anal. 16, 472-491 (1985).

[32] M.I. Weinstein, Lyapunov stability of ground states of nonlinear dispersive evolution equations, Comm. Pure Appl. Math. 39, 51-68 (1986).

[33] E. Yanagida, Uniqueness of positive radial solutions of $\Delta u+g(r) u+h(r) u^{p}=0$ in $\mathbb{R}^{n}$, Arch. Rat. Mech. Anal. 115, 257-274 (1991).

Anne De Bouard

Laboratoire de Mathématiques

Université de Paris-Sud

F-91405 Orsay

France

email: Anne.debouard@math.u-psud.fr

Reika Fukuizumi

Department of Mathematics

Hokkaido University

Sapporo 060-0810

Japan

email: reika@math.sci.hokudai.ac.jp

Communicated by Bernard Helffer

submitted 14/07/04, accepted 28/02/05 